\title{
GM1 improves neurofascin155 association with lipid rafts and prevents rat brain myelin injury after hypoxia-ischemia
}

\author{
Y.P. Zhang ${ }^{1,2}$, Q.L. Huang ${ }^{1}$, C.M. Zhao ${ }^{2}$, J.L. Tang ${ }^{3}$ and Y.L. Wang ${ }^{3}$ \\ ${ }^{1}$ Department of Neurosurgery, ${ }^{2}$ Department of Pediatrics, \\ ${ }^{3}$ Department of Pathology, Xinqiao Hospital, Third Military Medical University, \\ Chongqing, China
}

\begin{abstract}
White matter injury characterized by damage to myelin is an important process in hypoxic-ischemic brain damage (HIBD). Because the oligodendrocyte-specific isoform of neurofascin, neurofascin 155 (NF155), and its association with lipid rafts are essential for the establishment and stabilization of the paranodal junction, which is required for tight interaction between myelin and axons, we analyzed the effect of monosialotetrahexosyl ganglioside (GM1) on NF155 expression and its association with lipid rafts after HIBD in Sprague-Dawley rats, weighing 12-15 g, on day 7 post-partum (P7; $\mathrm{N}=20$ per group). HIBD was induced on P7 and the rats were divided into two groups: one group received an intraperitoneal injection of $50 \mathrm{mg} / \mathrm{kg} \mathrm{GM} 1 \mathrm{three}$ times and the other group an injection of saline. There was also a group of 20 sham-operated rats. After sacrifice, the brains of the rats were removed on P30 and studied by immunochemistry, SDS-PAGE, Western blot analysis, and electron microscopy. Staining showed that the saline group had definite rarefaction and fragmentation of brain myelin sheaths, whereas the GM1 group had no obvious structural changes. The GM1 group had 1.9-2.9-fold more GM1 in lipid rafts than the saline group (fraction 3-6; all $P<0.05$ ) and $0.5-2.4-$ fold higher expression of NF155 in lipid rafts (fraction 3-5; all P < 0.05). Injection of GM1 increased the content of GM1 in lipid rafts as well as NF155 expression and its lipid raft association in HIBD rat brains. GM1 may repair the structure of lipid rafts, promote the association of NF155 (or other important proteins) with lipid rafts, stabilize the structure of paranodes, and eventually prevent myelin sheath damage, suggesting a novel mechanism for its neuroprotective properties.
\end{abstract}

Key words: Cerebral hypoxia; Cerebral ischemia; Ganglioside; Myelin; Neurofascin 155

\section{Introduction}

Perinatal hypoxia-ischemia $(\mathrm{HI})$ is a common cause of neonatal brain damage that seriously impacts cerebral maturation and contributes to long-term neurological disabilities, including mental retardation, cerebral palsy, and epilepsy. The maladaptive neurobiological response can be severe and result in deep cerebral white matter injury and substantial neuronal loss. The primary characteristic of white matter injury after perinatal hypoxic-ischemic brain damage (HIBD) is damage to myelin. Myelinated axons are commonly divided into four functional regions: nodes of Ranvier, paranodes, juxtaparanodes, and internodes (1). The ion channels and membrane proteins of the nodes and juxtaparanodes are separated by specialized paranodal junctions, which require an adhesion complex that consists of the glial-specific 155-kDa isoform of neurofascin (NF155) at the end loops of the oligodendrocytes and Caspr and contactin of the axonal membrane. The correct assembly of paranodal junctions is vital to the integrity of the node and juxtaparanodal regions, permitting fast neurotransmission along myelinated fibers (1-3).

NF155 is a member of the immunoglobulin L1 subfamily. It is specifically localized in the axo-oligodendroglial paranodal junctions and plays important roles in myelin sheath development by preserving the structure and function of myelin sheaths (4), especially in the establishment of the paranodal junction $(2,5)$. NF155 promotes the aggregation of Caspr and contactin at paranodal junctions and has a "pioneering" role in axo-glial junction formation and node assembly (2). The role of NF155 in white matter diseases has been an area of increased interest in recent years. NF155 expression decreases as the result of increased degradation in multiple sclerosis (MS), resulting in destruc-

Correspondence: Q.L. Huang, Department of Neurosurgery, Xinqiao Hospital, Third Military Medical University, 183 Xinqiao Main Street, Shapingba District, Chongqing 400037, China. Fax: +86-23-6875-5602. E-mail: hqlmdxq@yahoo.com

Received November 4, 2010. Accepted May 11, 2011. Available online June 13, 2011. Published June 13, 2011. 
tion of the paranodal complex and disturbing the insulation of myelin that is vital for fast neurotransmission in the central nervous system (CNS) (6).

Gangliosides are a series of sphingolipids located mainly in the CNS and are essential for nervous system development and for the integrity of axons and myelin. Exogenous gangliosides show a neuroprotective effect in many cases of brain injury. Monosialotetrahexosyl ganglioside (GM1) is highly localized in lipid rafts $(7,8)$. Lipid rafts, or specialized membrane lipid microdomains, have been suggested to participate in a mechanism by which signaling, trafficking, and the assembly of adhesion complexes take place (9). Raft lipids are important in myelination because they maintain the structure of the paranodal loops $(10,11)$. NF155 is mainly located at the lipid raft of paranodes. The interaction between oligodendroglial NF155 and axonal ligands results in cross-linking, stabilization, and formation of paranodal lipid raft assemblies $(12,13)$. In some CNS white matter diseases, such as MS, the association of NF155 with rafts in the brain or spinal cord is reduced, thereby reducing the electrical isolation of myelin (14). We investigated how the main components of paranodal lipid rafts, GM1 and NF155, change quantitatively in HIBD rat. Furthermore, we determined whether exogenous GM1 can increase the association of GM1 and NF155 with lipid rafts in order to know whether the neuroprotective effect of GM1 is related to lipid raft repair.

\section{Material and Methods}

\section{Experimental animals}

Sprague-Dawley rats weighing $12-15 \mathrm{~g}$ were obtained on postpartum day 7 (P7) from the Experimental Animal Center, Third Military Medical University (Chongqing, China). The day of birth was considered to be P0. All experimental procedures were conducted in accordance with local guidelines on the ethical use of animals and with the National Institutes of Health "Guide for the Care and Use of Laboratory Animals" (NIH publication No. 80-23, revised 1996). The perinatal rat model of $\mathrm{HI}$ was used as described by Levine (15). Briefly, after P7 rats were anesthetized with ether, the left common carotid artery was cut between double ligatures. After surgery, the pups were allowed to recover for 1-1.5 $\mathrm{h}$. The rat pups were then placed in a humidified container maintained at $37^{\circ} \mathrm{C}$. $\mathrm{HI}$ was induced by perfusing the container with humidified $8 \%$ oxygen in a nitrogen gas mixture for $2 \mathrm{~h}$. After hypoxic exposure, the pups were returned to their biological dams. Sham-operated rat pups were randomly chosen from the same litters as the treated rats and were not subjected to common carotid artery ligation or to a period of hypoxia. After $\mathrm{HI}$ was induced, the neonatal rats were randomly divided into two groups: GM1 group $(\mathrm{N}=20)$ and Saline group $(N=20)$. The $\mathrm{GM} 1$ group received an intraperitoneal (ip) injection of $100 \mu \mathrm{L}$ GM1 (TRB Pharma, Argentina) at $50 \mathrm{mg} / \mathrm{kg}$ at 0,24 , and $48 \mathrm{~h}$ after $\mathrm{HI}$, three times in all. The Saline group received isovolumic normal saline ip injections at the same times. Rats from both genders were included in the experiments. The two groups were reared by dams in the same environment, that also included rats from the sham-operated (Sham) group $(N=20)$, which did not receive any injections.

\section{Myelin staining}

The structure of the myelin sheath in the rat brain is well developed at P30, and the development of the myelin sheath in rats at P30 is similar to that of humans at the ages of 2-5 years (16). We, therefore, chose P30 rats to detect damage to the myelin sheaths. Rats were deeply anesthetized and perfused with cold phosphate-buffered saline (PBS), followed by $4 \%$ buffered paraformaldehyde through the left ascending aorta. Brains were removed and the middle $1 / 3$ of brains were post-fixed in $4 \%$ paraformaldehyde and embedded in paraffin. Paraffin-embedded $4-\mu \mathrm{m}$ thick samples were rinsed in distilled water after deparaffination and then transferred through 95\% ethanol to a $0.1 \%$ solution of luxol fast blue (LFB; Sigma, USA) in $95 \%$ ethanol and $0.05 \%$ acetic acid. After staining for 16 $\mathrm{h}$ at $60^{\circ} \mathrm{C}$, sections were washed with distilled water, differentiated in $0.05 \%$ aqueous lithium carbonate followed by $70 \%$ ethanol, washed, and counterstained with nuclear fast red before standard mounting, dehydrating, and coverslipping. Staining on frontal plane 15 of the left hemisphere according to the rat brain atlas was observed (17).

\section{Electron microscopy}

At $\mathrm{P} 30,5$ rats were randomly selected from each group and perfused with cold PBS, followed by $2 \%$ buffered paraformaldehyde and $2 \%$ glutaraldehyde through the left ascending aorta. After sacrifice, brains were removed and white matter $(1 \times 1 \times 1 \mathrm{~mm})$ from a similar area of the left hemisphere above left paracele was sampled from an area $<1 \mathrm{~mm}$ posterior from Bragma site and $>1 \mathrm{~mm}$ from midline (17) and post-fixed in $3 \%$ glutaraldehyde $\left(0-4^{\circ} \mathrm{C}\right)$ and incubated with $1 \%$ osmic acid for $3 \mathrm{~h}$ after rinsing. The samples were dehydrated with acetone and embedded in epoxy resin. Ultrathin sections $(60 \mathrm{~nm})$ were then cut from the resin-embedded samples and stained with uranyl acetate and lead citrate prior to examination by transmission electron microscopy.

\section{Immunohistochemistry}

Immunohistochemical characterization of the tissue was performed on 4- $\mu \mathrm{m}$ thick sections of paraffin-embedded samples collected from a similar area as for myelin staining. Briefly, sections were microwaved for antigen retrieval and pretreated with $0.3 \% \mathrm{H}_{2} \mathrm{O}_{2}$. Subsequently, the sections were blocked with goat serum and incubated in a primary antibody solution containing rabbit anti-myelin 
basic protein (MBP) antibody (Maixin Biotechnology, Fuzhou, China) overnight at $4^{\circ} \mathrm{C}$. After washing, the samples were incubated in a secondary antibody solution consisting of sheep anti-rabbit (1:200, Zhongshan Biotechnology, China) for $1 \mathrm{~h}$ at room temperature. Finally, the sections were incubated in HRP-streptavidin (1:200, Zhongshan Biotechnology) for $1 \mathrm{~h}$ at room temperature, and the color reaction was developed with diaminobenzidine (DAB) and $\mathrm{H}_{2} \mathrm{O}_{2}$. After counterstaining with $0.1 \%$ cresyl violet, sections were dehydrated, coverslipped, and analyzed using a conventional microscope (DMIRB; Leica, Germany).

\section{Protein extraction}

Frozen rat left hemispheres were homogenized and added to lysis buffer (50 mM Tris, pH 7.5, $150 \mathrm{mM} \mathrm{NaCl}$, $1 \%$ Nonidet NP-40, 10\% glycerol, 2 mM 4-(2-aminoethyl)benzenesulfonyl fluoride, $1 \mathrm{mg} / \mathrm{mL}$ leupeptin, $2 \mathrm{mg} / \mathrm{mL}$ aprotinin, and $2 \mathrm{mg} / \mathrm{mL}$ pepstatin), gently sonicated, incubated on ice for $30 \mathrm{~min}$, and centrifuged at 12,000 $\mathrm{g}$ for $15 \mathrm{~min}$. The extracts were normalized for equal amounts of total protein measured by the bicinchoninic acid (BCA) method. Fifty micrograms protein from each sample was analyzed by SDS-PAGE and Western blot.

\section{Detergent extraction and gradient centrifugation}

Detergent extraction and gradient centrifugation were performed as described by Maier et al. (14). Briefly, rat brain hemisphere homogenates were resuspended in $100 \mathrm{mg} / 400 \mu \mathrm{L}$ TNE buffer (50 mM Tris, $150 \mathrm{mM} \mathrm{NaCl}, 5$ mM EDTA, pH 7.3), containing 1\% TX100 and protease inhibitors (Roche, Germany), pressed 25 times with a glass tube/Teflon pestle, and incubated for $30 \mathrm{~min}$ on ice with occasional mixing. To determine the extent of association of proteins with lipid rafts, $250 \mu \mathrm{L}$ of the extract was mixed with $500 \mu \mathrm{L} 60 \%$ OptiPrep (Axis-shield, Norway) and loaded at the bottom of a centrifuge tube (CP-80wx, Hitachi, Japan), with 2.25 mL 30\% OptiPrep in TNE buffer in the middle and $10 \%$ OptiPrep in TNE buffer layered on top. After centrifugation for $17 \mathrm{~h}$ at 200,000 $g$, the gradients were formed in seven $750-\mu \mathrm{L}$ fractions. Fractions were freeze-dried and redissolved. Then, equal volumes of each fraction were analyzed by SDS-PAGE and Western blot.

\section{SDS-PAGE and Western blotting}

Protein samples or gradient fractions were mixed with SDS sample buffer, heated for 2 min at $98^{\circ} \mathrm{C}$, and separated by SDS-PAGE. Proteins were transferred to PVDF membranes. The membranes were blocked with $5 \%$ nonfat milk and incubated overnight with primary anti-NF155 antibodies (1:300, Abcam, UK) or anti-GM1 antibody $(1: 150, \mathrm{Abcam})$ at $4^{\circ} \mathrm{C}$, followed by incubation with the suitable HRP-conjugated secondary antibody for $1 \mathrm{~h}$. Signals on Western blots were detected by enhanced chemiluminescence (Amersham, UK). Densitometric analysis was performed by using the Image-Pro Plus software (Media Cybernetics, USA).

\section{Statistical analysis}

Data are reported as means \pm SD. To determine significant differences among the three groups, statistical analysis was performed by one-way analysis of variance (ANOVA) with Bonferroni's corrections. Differences were considered to be statistically significant when $\mathrm{P}<0.05$. Statistical analyses were performed using SPSS version 15.0 (SPPS Inc., USA).

\section{Results}

\section{Morphological changes in gross brain and myelin sheath (LFB and MBP staining)}

A bilaterally symmetric gross morphology and smooth surface were observed in the brains of the Sham group (Figure 1A). In contrast, the brains of the Saline group (Figure 1E) showed significant left cerebral hemisphere atrophy, and an occasional focus of malacia (Figure 1B). Brains of the GM1 group (Figure 1I) were similar to those of the Sham group, with no atrophy and no focus of malacia. In LFB staining and anti-MBP immunohistochemical staining of brain coronal sections, the cerebral cortex and white matter of Sham group brains (Figure 1B-D) were clearly distributed and bilaterally symmetric (Figure 1A). The corpus callosum (CC), cingulate $(\mathrm{Cg})$, external capsule (EC), and internal capsule all showed LFB-positive (blue-colored) or MBP-positive (brown-colored) myelin sheath staining. Blue or brown filamentous staining was detected in the inner cortex, especially in the lateral $\mathrm{Cg}$. In the Saline group, the right cerebral hemisphere showed staining similar to that of the Sham group brains; however, the CC and EC of the left cerebral hemisphere showed clear rarefaction and fragmentation, with distension of blood vessels (Figure $1 \mathrm{~F}-\mathrm{H})$. The brain sections of the GM1 group exhibited a staining pattern intermediate between that of the Sham and Saline groups, without obvious structural changes (Figure 1J-L). These observations indicate that the white matter was damaged after $\mathrm{HI}$ and that GM1 attenuated the damage in the myelin sheath.

\section{Western blot analysis of the distribution of GM1 in lipid rafts of rat brain}

The distribution of endogenous GM1 in lipid rafts of the left cerebral hemispheres of all three groups was detected by Western blot analysis after extraction of lipid rafts by gradient centrifugation (Figure 2). The amount of GM1 in lipid rafts was significantly lower in the Saline group compared to the Sham group $(P<0.05)$ and was much higher in the GM1 group compared to the Saline group (fraction 3: $73.37 \pm 5.12$ vs $18.75 \pm 2.04$; fraction 4 : $103.07 \pm 16.65$ vs $36.08 \pm 3.02$; fraction 5 : $92.97 \pm 16.28$ vs $31.09 \pm 5.86$; fraction 6 : $7.14 \pm 2.30$ vs $2.48 \pm 0.89$; all 
Gross appearance

\section{LFB staining}

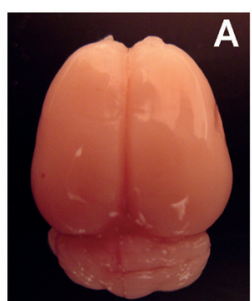

Sham
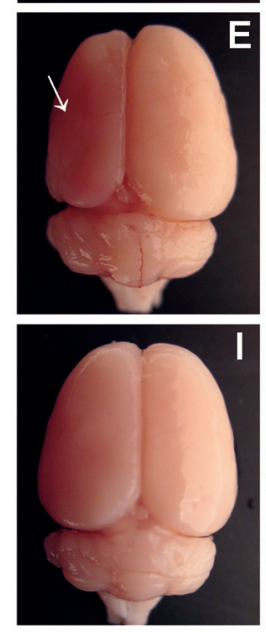
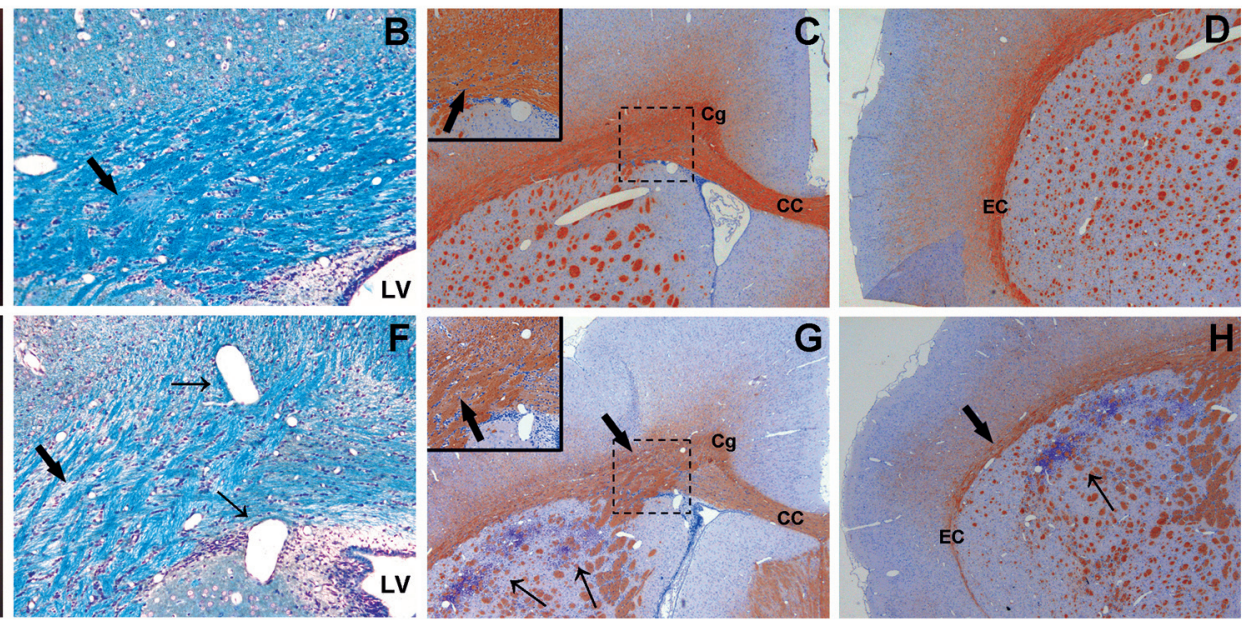

G
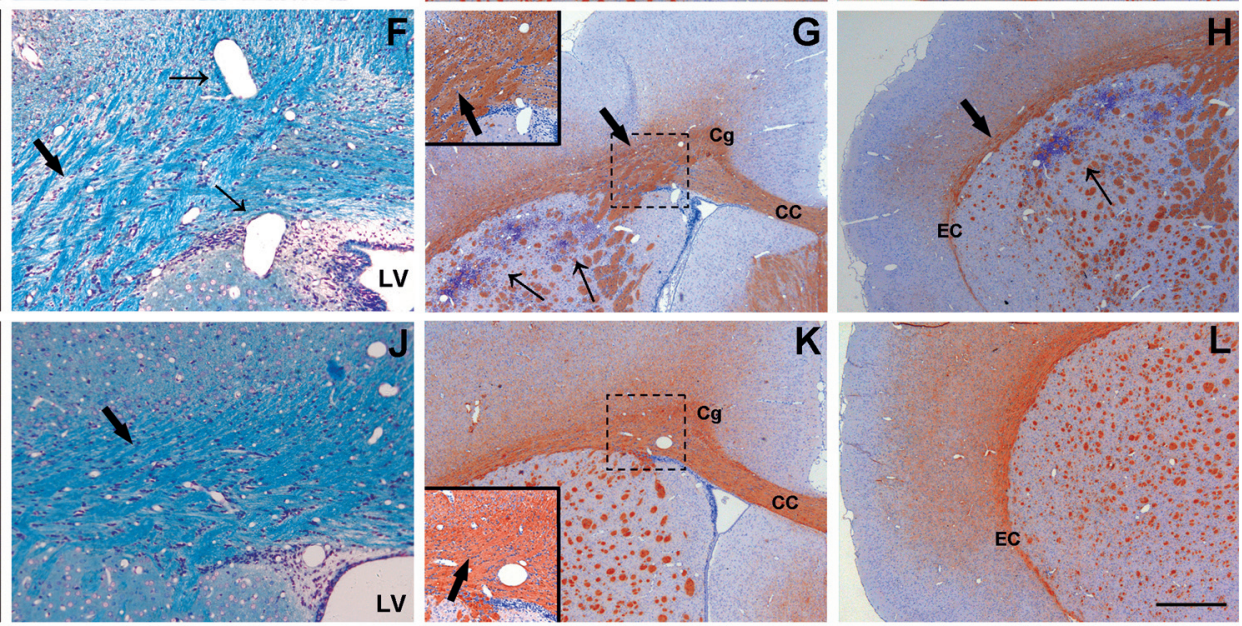

Figure 1. Morphological changes in gross brain ( $A, E, I)$ and myelin sheath detected by luxol fast blue (LFB) (B, F, and J) and myelin basic protein (MBP) (C, D, G, H, K, and L) staining in the three groups of P30 rats. After hypoxic-ischemic brain damage was induced, one group received an intraperitoneal injection of saline (Saline group) and another group was injected with monosialotetrahexosyl ganglioside (GM1; GM1 group). Sham-operated rats served as control (Sham group). The gross morphology of the Saline group brains (E) showed significant left cerebral hemisphere atrophy (the white arrow indicates the atrophied brain). Brains from the GM1 group (I) were similar to those from the Sham group (A). LFB staining in the corpus callosum (CC) and cingulum (Cg) showed compact myelin sheaths in the Sham group (thick black arrow; B) and clear structural abnormalities of rarefaction and fragmentation (thick black arrow) with distension of the blood vessels (thin black arrows) in the Saline group (F); whereas GM1 group (J) brains were similar to those of the Sham group rats, with improved myelin sheath density (thick black arrow). Immunohistochemical staining of MBP showed structural abnormalities of rarefaction and fragmentation in the left CC and external capsule (EC; thick black arrows) in the Saline group $(G, H)$, with infiltration of inflammatory cells in the left cerebral hemisphere (thin black arrows; $G, H)$. A high magnification view shows abnormalities of myelin in the $\mathrm{CC}$ and $\mathrm{Cg}$. The staining pattern of the left CC and EC of the GM1 groups $(\mathrm{K}, \mathrm{L})$ was similar to that of the Sham group (C, D), without structural abnormalities. Scale bars: $3 \mathrm{~mm}$ for A, E, and I; $125 \mu \mathrm{m}$ for B, F, and J, and $500 \mu \mathrm{m}$ for C, D, G, $\mathrm{H}, \mathrm{K}$, and L. For Panels C, G, and K, the area framed with dashed lines was zoomed in 1.8-fold and shown in insets with thick black arrows indicating the changes in myelin sheath.

$P<0.05)$, which indicates that the amount of endogenous GM1 in the lipid rafts of rat brains was reduced after HI and that supplementation with exogenous GM1 significantly increase the content of GM1 in lipid rafts.

\section{Effects of $\mathrm{HI}$ and GM1 intervention on NF155 levels in brains and in lipid rafts}

The expression level of NF155 was examined in brain tissue and lipid rafts of the left cerebral hemispheres of each group. The $200-k D a$ NF155 protein was detected in both protein extracts and lipid rafts (the protein band was less mobile than expected on the SDS-PAGE gel due to glycosylation). The Saline group showed the lowest amount of NF155 in brain tissue and the NF155 level in the Saline group was significantly lower than that in the Sham group (Figure 3A) $(P<0.05)$. The NF155 level in the GM1 group was higher than that in the Saline group but lower than that in the Sham group, although the differences were not statistically significant. Among the three groups, the Saline group showed the lowest level of NF155 in lipid rafts (Figure 3B). The lipid raft NF155 level in the GM1 group was significantly higher than that of the Saline group (fraction 3: $52.10 \pm 4.54$ vs $24.53 \pm 5.55$; fraction 4 : $80.19 \pm 3.84$ vs $53.45 \pm 7.97$; fraction $5: 54.88 \pm 3.27$ vs $16.15 \pm 2.20$; all $P<0.05)$. The fold values for fractions 3,4 , and 5 were $0.56,0.52$, and 0.24 , respectively, in the Saline group and $1.19,0.77$, and 

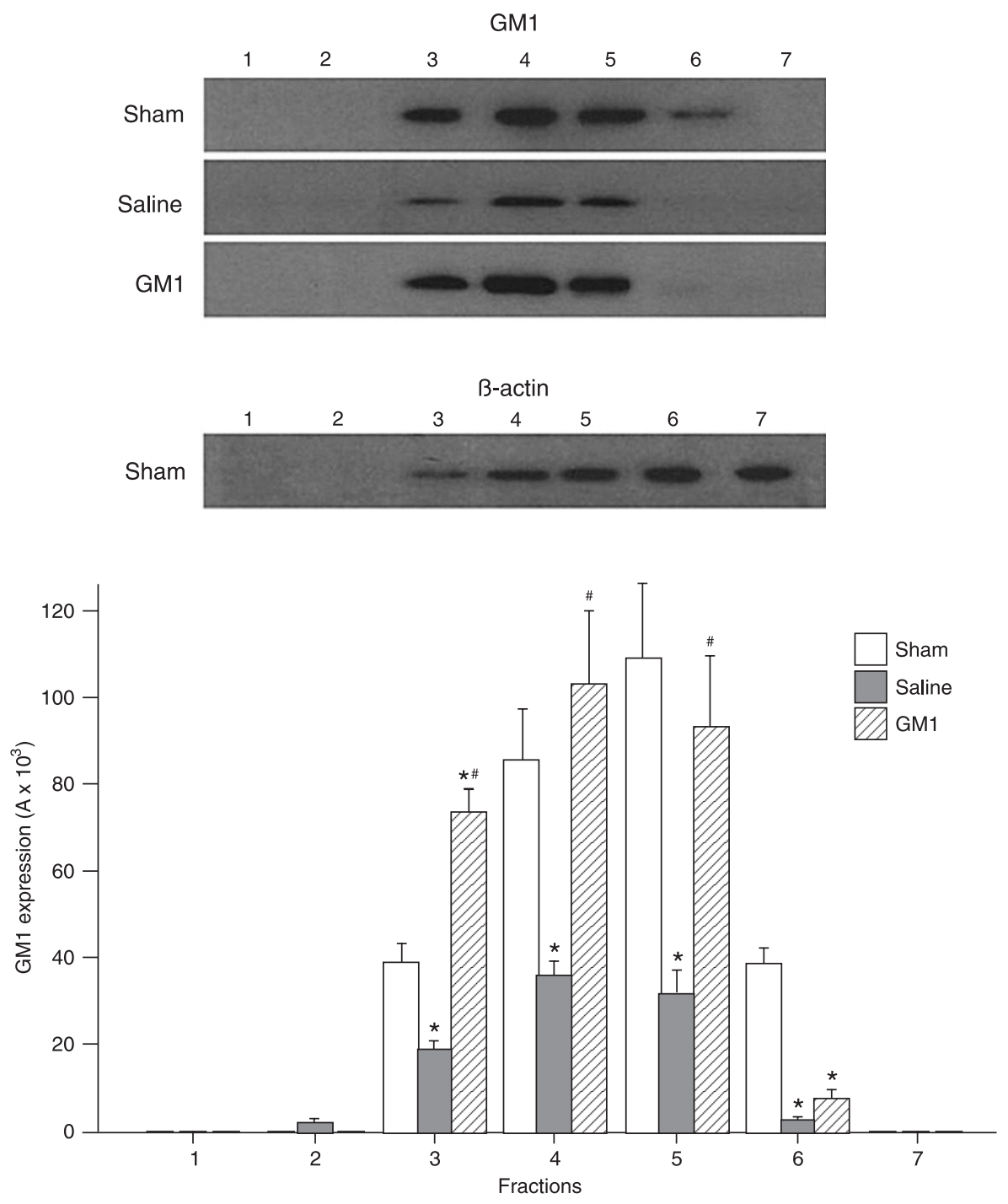

Figure 2. Western blot analysis of monosialotetrahexosyl ganglioside (GM1) in lipid rafts from left cerebral hemispheres of each rat group at postpartum day 30 (P30). The Western blot (lanes 1-7) showed the bands of GM1 in 4 of the 7 fractions prepared by OptiPrep gradient centrifugation. Raft fractions (fractions 3-5) are indicated. $A=$ absorbance. GM1 expression was significantly lower in lipid rafts of the Saline group compared to the Sham group $\left({ }^{*} P<0.05\right)$, and the expression of GM1 in lipid rafts of the GM1 group was much higher than that in the Saline group (\#P $<0.0 .05$, one-way analysis of variance (ANOVA) with Bonferroni's corrections in post hoc tests).

0.82 , respectively, in the GM1 group. These results indicate that $\mathrm{HI}$ reduced the NF155 level in rat brains, particularly in lipid rafts, and that GM1 increased the expression of NF155 in the brain, particularly in lipid rafts.

\section{Electron microscopy findings}

Figure 4A shows that in the Sham group the myelin sheath retained its integrity and was compact. Figure 4B demonstrates that in the Saline group there was rarefaction of the myelin sheath with obvious separation between lamel- lae and a disordered structure. In addition, shrinkage of the axoplasm was also observed in the Saline group (Figure 4C). Figure 4D from a rat in the GM1 group shows that, compared to the Saline group, the myelin sheath was compact and there was less separation between lamellae.

\section{Discussion}

NF155 is an important protein of oligodendroglial origin localized at the paranode, which plays an important role in 
A

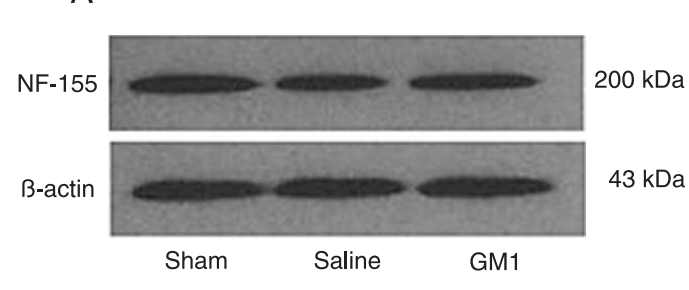

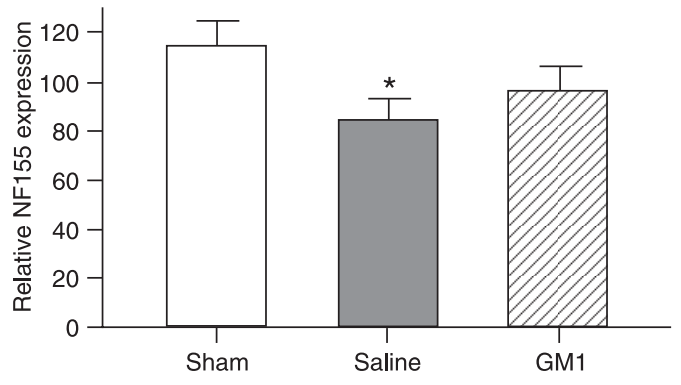

B
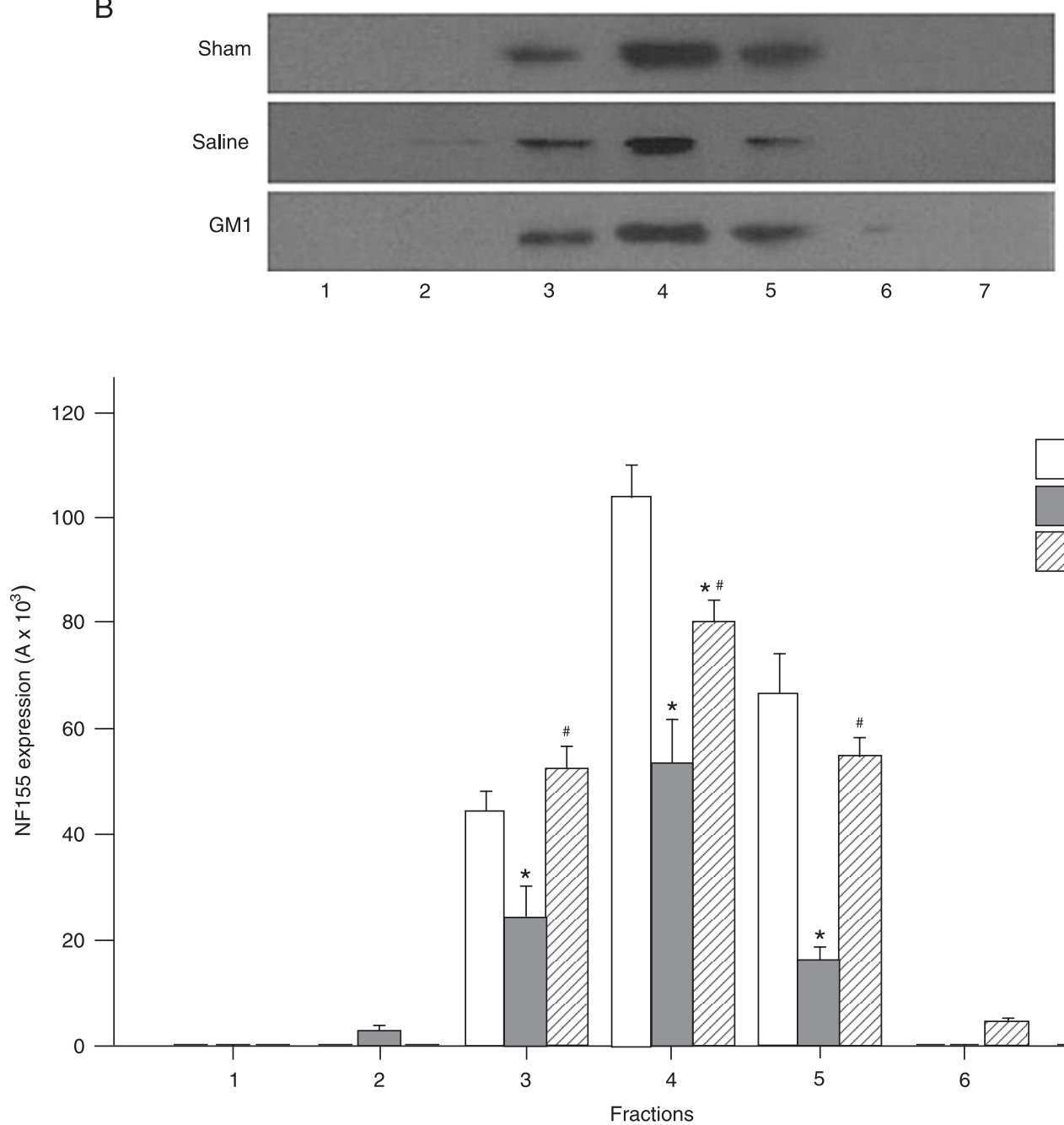

Figure 3. Western blot analysis of neurofascin 155 (NF155) expression in the brain tissue and lipid rafts of rat left cerebral hemispheres from each group at postpartum day 30 (P30). A, NF-155 was present as a 200-kDa band in the lanes, which corresponded to a higher molecular mass than expected due to glycosylation. The bar graph on the right shows that NF155 content was significantly lower in the Saline group compared to the Sham group and was higher in the GM1 group injected with monosialotetrahexosyl ganglioside (GM1) than in the Saline group. Relative expression was calculated by normalization of the absorbance (A) of NF155/A to that of control ( $\beta$-actin). B, Western blot analysis of NF155 in lipid rafts showed that the NF155 content of the Saline group was significantly lower than in the Sham group and was higher in the GM1 group than in the Saline group. Lanes 1-7 in the Western blot show the bands of NF155 in 7 fractions prepared by OptiPrep gradient centrifugation. ${ }^{*} \mathrm{P}<0.05$ vs Sham group; ${ }^{\#} \mathrm{P}<0.05$ vs saline group (one-way analysis of variance (ANOVA) with Bonferroni's corrections in post hoc tests). 

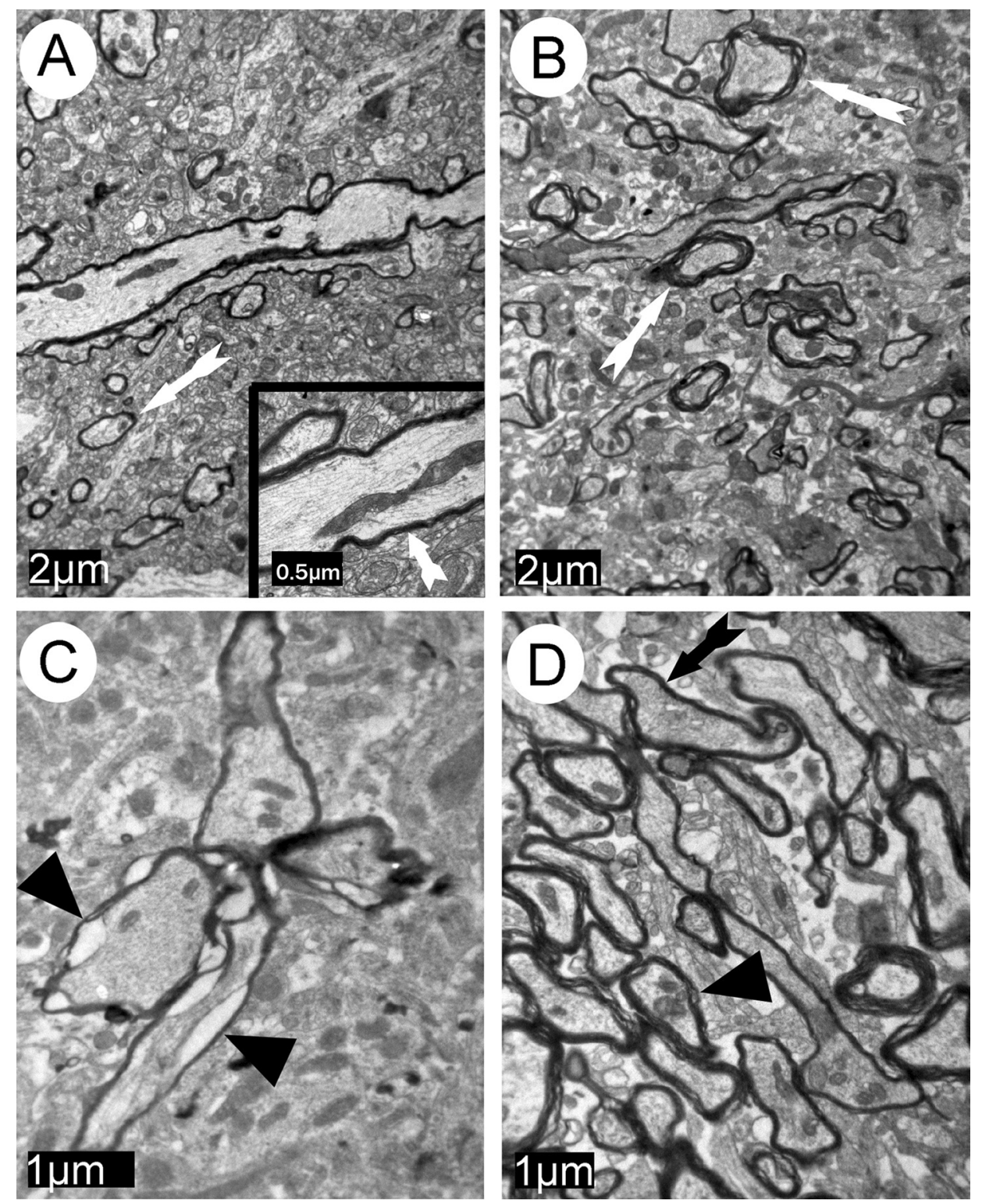

Figure 4. Electron microscopy images of the brain. $A$, Myelin sheaths in a Sham group rat retained their integrity and were compact (white arrow). Higher magnification is shown in the inset in the lower right corner. $B$, Myelin sheath in a Saline group rat after hypoxic-ischemic brain damage (HIBD) was characterized by rarefaction and there was obvious separation between lamellae with disordered structure, as indicated by the white arrows. $C$, Axoplasm shrinkage can be seen in an image from a Saline group rat at higher magnification (black arrowheads). $D$, Image from a rat injected with monosialotetrahexosyl ganglioside (GM1 group) after HIBD shows that, compared to the Saline group, the myelin sheaths are compact and there is less separation between lamellae, as indicated by the black arrow/arrowhead.

myelin sheath development by preserving the structure and function of myelin sheaths. Researchers have studied the role of NF155 in autoimmune diseases associated with CNS white matter and have observed changes in its expression and distribution in these diseases. Auto-antibodies against
NF155 have been identified in brain tissues from MS patients (13). In the white matter lesion areas in MS patients, the expression level of NF155 was decreased, and the expression of degraded NF155 proteins was increased. Abnormal ion channel distribution in axons was observed (6). Myelin 
sheath insulation is affected by the significantly decreased content of NF155 in lipid rafts and by the damage to the protein complex structure in the paranodal area. This phenomenon was also observed in experimental animal models of autoimmune encephalomyelitis (18). These observations strongly suggest that NF155 and its association with the lipid raft play an important role in white matter-associated autoimmune disorders. We found that the expression of NF155 significantly decreased in HIBD rat brains and that its distribution in lipid rafts also decreased, indicating that NF155 participates in the white matter damage observed in HIBD.

GM1 plays an important role in nervous system development, specifically myelin sheath development. A high level of GM1 is found in white matter, especially in myelin sheaths (19). GM1 is mainly localized in lipid rafts and is widely used as a marker of lipid rafts (20). In mutant mice lacking GM1 and GD1a in the CNS, some paranodal oligodendrocyte loops failed to attach to the axolemma to form myelin sheaths, the expression of Caspr and NF155 was reduced, and the content of these proteins in lipid rafts was also reduced (7). A significant reduction of several gangliosides was observed after $\mathrm{HI}$ (21), which may contribute to the structural changes in the myelin sheaths. In our study, we found that HI led to a decrease of GM1 and NF155 in lipid rafts, suggesting that during the perinatal stage $\mathrm{HI}$ may destroy the structure of lipid rafts, change the lipid and protein composition of lipid rafts in paranodes, decrease paranodal junction stability, and induce abnormal myelin development.

Exogenous GM1 has displayed neuroprotective effects in many cases of nervous system injury, which may be due to its ability to inhibit excitatory amino acid release (22), its antagonist effects on excitatory amino acid receptors (23), or its antioxidant activities $(24,25)$. We found that ip injection of GM1 clearly increased the distribution of GM1 and NF155 in lipid rafts. Electron microscopy showed that injection of GM1 helped preserve the structure of myelin sheaths, as the myelin sheaths in the GM1 group appeared to be more

\section{References}

1. Poliak S, Peles E. The local differentiation of myelinated axons at nodes of Ranvier. Nat Rev Neurosci 2003; 4: 968980.

2. Sherman DL, Tait S, Melrose S, Johnson R, Zonta B, Court FA, et al. Neurofascins are required to establish axonal domains for saltatory conduction. Neuron 2005; 48: 737-742.

3. Salzer JL. Polarized domains of myelinated axons. Neuron 2003; 40: 297-318.

4. Koticha D, Babiarz J, Kane-Goldsmith N, Jacob J, Raju K, Grumet M. Cell adhesion and neurite outgrowth are promoted by neurofascin NF155 and inhibited by NF186. Mol Cell Neurosci 2005; 30: 137-148.

5. Charles P, Tait S, Faivre-Sarrailh C, Barbin G, Gunn-Moore compact with less obvious separation between the myelin lamellae compared to the Saline group. Moreover, our results indicate that the neuroprotective effects of exogenous GM1 may result from its ability to increase the quantity of important lipids in paranodes, to repair the structure of lipid rafts, to promote the association of NF155 or other important paranode proteins with lipid rafts, and to protect these important proteins, thereby stabilizing the structure of paranodes, and eventually repairing the damage to the myelin sheath. Alternatively, the neuroprotective effect of GM1 might be related to the effect of GM1 on blood vessel dynamics. Furian et al. (26-28) have demonstrated in rats that GM1 induces vasodilation in the brain and that nitric oxide and potassium channels apparently play important roles in this effect. We found that blood vessels were distended in the Saline group and did not observe distension of blood vessels in the GM1 group. Further studies are needed to determine if the effect of GM1 on blood vessel dynamics plays any role in the neuroprotective effect of GM1.

The change in the association between NF155 and lipid rafts may therefore be involved not only in autoimmune myelin sheath degenerative diseases but also in developmental myelin sheath disorders. As a neuroprotective agent specifically present in myelin sheaths and lipid rafts, GM1 shows a neuroprotective effect by repairing the lipid raft when supplemented exogenously. The present results show the presence of a novel neuroprotective mechanism of GM1.

\section{Acknowledgments}

Research supported by the Key Scientific and Technological R\&D Program of the State Family Planning Commission (\#2007B070) whose project name is "Effect of Adverse perinatal factors on neonatal neurological development". We thank Dr. Chunqin Zhang and Dr. Haifeng Shu for excellent technical assistance, help with figure preparation, and helpful comments on the manuscript.
F, Denisenko-Nehrbass N, et al. Neurofascin is a glial receptor for the paranodin/Caspr-contactin axonal complex at the axoglial junction. Curr Biol 2002; 12: 217-220.

6. Howell OW, Palser A, Polito A, Melrose S, Zonta B, Scheiermann $C$, et al. Disruption of neurofascin localization reveals early changes preceding demyelination and remyelination in multiple sclerosis. Brain 2006; 129: 3173-3185.

7. Susuki K, Baba H, Tohyama K, Kanai K, Kuwabara S, Hirata $\mathrm{K}$, et al. Gangliosides contribute to stability of paranodal junctions and ion channel clusters in myelinated nerve fibers. Glia 2007; 55: 746-757.

8. Sheikh KA, Deerinck TJ, Ellisman MH, Griffin JW. The distribution of ganglioside-like moieties in peripheral nerves. 
Brain 1999; 122 (Part 3): 449-460.

9. Brown DA, London E. Structure and function of sphingolipidand cholesterol-rich membrane rafts. J Biol Chem 2000; 275: 17221-17224

10. Ishibashi T, Dupree JL, Ikenaka K, Hirahara Y, Honke K, Peles $\mathrm{E}$, et al. A myelin galactolipid, sulfatide, is essential for maintenance of ion channels on myelinated axon but not essential for initial cluster formation. J Neurosci 2002; 22: 6507-6514.

11. Dupree JL, Coetzee T, Suzuki K, Popko B. Myelin abnormalities in mice deficient in galactocerebroside and sulfatide. $J$ Neurocytol 1998; 27: 649-659.

12. Schafer DP, Bansal R, Hedstrom KL, Pfeiffer SE, Rasband $\mathrm{MN}$. Does paranode formation and maintenance require partitioning of neurofascin 155 into lipid rafts? J Neurosci 2004; 24: 3176-3185.

13. Schaeren-Wiemers N, Bonnet A, Erb M, Erne B, Bartsch U, Kern $F$, et al. The raft-associated protein MAL is required for maintenance of proper axon-glia interactions in the central nervous system. J Cell Biol 2004; 166: 731-742.

14. Maier O, Baron W, Hoekstra D. Reduced raft-association of NF155 in active MS-lesions is accompanied by the disruption of the paranodal junction. Glia 2007; 55: 885-895.

15. Levine S. Anoxic-ischemic encephalopathy in rats. $A m \mathrm{~J}$ Pathol 1960; 36: 1-17.

16. Rodier PM. Vulnerable periods and processes during central nervous system development. Environ Health Perspect 1994; 102 (Suppl 2): 121-124.

17. Paxinos G, Watson C. The rat brain in stereotaxic coordinates. San Diego: Academic Press, Inc.; 1997.

18. Maier O, van der Heide T, van Dam AM, Baron W, de Vries $\mathrm{H}$, Hoekstra D. Alteration of the extracellular matrix interferes with raft association of neurofascin in oligodendrocytes. Potential significance for multiple sclerosis? Mol Cell Neurosci 2005; 28: 390-401.

19. Yates AJ. Gangliosides in the nervous system during de- velopment and regeneration. Neurochem Pathol 1986; 5: 309-329.

20. Simons K, Ikonen E. Functional rafts in cell membranes. Nature 1997; 387: 569-572.

21. Ramirez MR, Muraro F, Zylbersztejn DS, Abel CR, Arteni NS, Lavinsky D, et al. Neonatal hypoxia-ischemia reduces ganglioside, phospholipid and cholesterol contents in the rat hippocampus. Neurosci Res 2003; 46: 339-347.

22. Phillis JW, O'Regan MH. GM1 ganglioside inhibits ischemic release of amino acid neurotransmitters from rat cortex. Neuroreport 1995; 6: 2010-2012.

23. Liu JR, Ding MP, Wei EQ, Huang JZ, Song $Y$, Ding Q, et al. Monosialoganglioside protected ischemic rat hippocampal slices through stabilizing expression of N-methyl-Daspartate receptor subunit. Acta Pharmacol Sin 2004; 25: 727-732.

24. Mahadik SP, Makar TK, Murthy JN, Ortiz A, Wakade CG, Karpiak SE. Temporal changes in superoxide dismutase, glutathione peroxidase, and catalase levels in primary and peri-ischemic tissue. Monosialoganglioside (GM1) treatment effects. Mol Chem Neuropathol 1993; 18: 1-14.

25. Fighera MR, Bonini JS, Frussa-Filho R, Dutra-Filho CS, Hagen ME, Rubin MA, et al. Monosialoganglioside increases catalase activity in cerebral cortex of rats. Free Radic Res 2004; 38: 495-500.

26. Furian AF, Oliveira MS, Royes LF, Fiorenza NG, Fighera MR, Myskiw JC, et al. GM1 ganglioside induces vasodilation and increases catalase content in the brain. Free Radic Biol Med 2007; 43: 924-932.

27. Furian AF, Oliveira MS, Magni DV, Souza MA, Bortoluzzi VT, Bueno LM, et al. L-NAME prevents GM1 gangliosideinduced vasodilation in the rat brain. Neurochem Int 2008; 53: 362-369.

28. Furian AF, Rattmann YD, Oliveira MS, Royes LF, Marques $M C$, Santos AR, et al. Nitric oxide and potassium channels mediate GM1 ganglioside-induced vasorelaxation. Naunyn Schmiedebergs Arch Pharmacol 2009; 380: 487-495. 